\title{
ESTIMACIÓN DE RENDIMIENTO EN EL CULTIVO DE CAÑA DE AZÚCAR (Saccharum officinarum) A PARTIR DE FOTOGRAMETRÍA CON VEHÍCULOS AÉREOS NO TRIPULADOS (VANT)*
}

\author{
Bryan Alemán-Montes ${ }^{1 * *}$, Carlos Henríquez-Henríquez ${ }^{2}$, \\ Tatiana Ramírez-Rodríguez ${ }^{3}$, Kenneth Largaespada-Zelaya ${ }^{4}$
}

Palabras clave: VANT; Caña de azúcar; fotogrametría; agricultura de precisión; estimación de rendimiento. Keywords: UAV; sugarcane; photogrammetry; precision agriculture; yield prediction.

Recibido: 20/04/2020

RESUMEN

Introducción. La estimación previa de variables de rendimiento en plantaciones de caña de azúcar (Saccharum officinarum), es importante para adaptar prácticas de manejo y aumentar la competitividad en la agroindustria azucarera costarricense. Objetivo. El objetivo de este estudio fue realizar estimaciones de variables de rendimiento en el cultivo de la caña de azúcar, por medio de la integración de fotografías aéreas, métodos fotogramétricos y determinaciones de campo. Materiales y métodos. Se utilizó un lote de una hectárea de caña de azúcar, propiedad de la cooperativa CoopeCañita R.L. (Turrialba, Costa Rica), con 5 años de producción y sembrado con la variedad B76-256. La evolución espacio-temporal del crecimiento del cultivo se midió de mayo a noviembre del

\footnotetext{
* Este proyecto se desarrolló bajo el convenio entre el Instituto Nacional de Fomento Cooperativo (INFOCOOP) y la Universidad de Costa Rica (UCR) en el Centro de Investigaciones Agronómicas (CIA), en cumplimiento de los objetivos de los proyectos VI-733-B5-A11 y VI-733-B5-508.

** Autor para correspondencia. Correo electrónico bryan.aleman@ucr.ac.cr

1 Universidad de Costa Rica, Centro de Investigaciones Agronómicas y Escuela de Geografía, Costa Rica.

(iD) 0000-0003-4349-2255.
}

Aceptado: 18/06/2020

\section{ABSTRACT}

\begin{tabular}{lrr}
\multicolumn{1}{c}{ Yield estimation of } & sugarcane \\
(Saccharum & officinarum) & from \\
photogrammetry with unmanned aerial
\end{tabular} vehicles (UAV). Introduction. The estimation of yield variables in sugarcane (Saccharum officinarum) previous to its harvest is important to adapt management practices and to increase the competitiveness of the Costa Rican sugar agroindustry. Objective. The aim of this study was to carry out an estimation of different yield variables in sugarcane through the integration of aerial photos, photogrammetric methods, and calibration of the processed image outputs against measured field data. Materials and methods. A plot with a 5-years old ratoon of the variety B76-256 of sugar cane from CoopeCañita R.L. (Turrialba, Costa Rica) was used for the

\footnotetext{
2 Universidad de Costa Rica, Centro de Investigaciones Agronómicas y Sede del Atlántico, Costa Rica. (D) 0000-0003-1487-4246.

3 Universidad de Costa Rica, Centro de Investigaciones Agronómicas y Escuela de Geografía, Costa Rica. (iD) 0000-0001-8598-1247.

4 Universidad de Costa Rica, Centro de Investigaciones Agronómicas y Sede del Atlántico, Costa Rica. (D) 0000-0003-4651-443X.
} 
2017, por medio de 6 vuelos con un VANT, el cual portaba una cámara fotográfica CoolPix A de 16,2 megapíxeles. Se realizó el procesamiento fotogramétrico y se obtuvieron los Modelos Digitales de Superficie (DSM). Estas variables permitieron estimar la altura y el volumen del cultivo. Las estimaciones se correlacionaron con diferentes variables de rendimiento como toneladas métricas de caña por hectárea (TMH), rendimiento de kilogramos de azúcar por tonelada (RKA), total de kilogramos de azúcar (TKA), total de kilogramos de miel (TKM) y rendimiento de kilogramos de miel (RKM) de la zafra 2017-2018. Resultados. La relación entre las TMH obtenidas en campo y las estimadas a partir del modelo de regresión lineal creado con la altura del cultivo fue de un $\mathrm{R}^{2}$ de 0,83 . El error absoluto de la estimación de rendimiento, a partir de altura de planta, fue de 3,7 ton.ha ${ }^{-1}$, el error residual estándar de 6,3 ton.ha ${ }^{-1}$ y el RMSE de 5,63 ton.ha ${ }^{-1}$. Conclusión. Según los resultados, la mejor fecha para la estimación TMH fue aproximadamente 3 meses antes de cosecha. No se encontró relación entre las estimaciones fotogramétricas y la variable RKA.

\section{INTRODUCCIÓN}

La estimación del rendimiento de algunos cultivos, así como el análisis de su variabilidad espacial y temporal, se puede realizar mediante el uso de diferentes herramientas geoespaciales, como por ejemplo, las imágenes satelitales, apoyadas en técnicas fotogramétricas (Shunling 2004, Marcos et al. 2016, Som-ard et al. 2018). Al utilizar esas herramientas se pretende facilitar el monitoreo espacial y temporal de las plantaciones para tener así, una respuesta rápida y oportuna, en procura de tomar las mejores decisiones (Oshunsanya y Aliku 2016). calibration. The spatial and temporal evolution of crop growth was monitored from May to November 2017 using an Unmanned Aerial Vehicle (UAV) that carried a 16.2-megapixel CoolPix A camera during 6 flights. The photogrammetric processing of the images and the generation of Digital Surface Models (DSM) allowed the estimation of plant height and crop volume. The estimations were correlated with different yield variables as metric tons of fresh biomass per hectare (TMH), kilograms of sugar per ton of fresh biomass (RKA), total kilograms of sugar per hectare (TKA) total kilograms of molasses per hectare (TKM) and yield of kilograms of molasses per ton of fresh biomass (RKM) of the 2017-2018 harvest. Results. The relationship between field-measured yield and the estimated values of plant height was $\mathrm{R}^{2}=0.83$. The absolute error for yield estimation using the photogrammetric obtained plant height was 3.7 ton.ha ${ }^{-1}$, its residual error was about 6.3 ton.ha $^{-1}$ and the RMSE was 5.63 ton.ha ${ }^{-1}$. Conclusion. According to the results, the best time to predict the sugarcane yield (TMH) through UAV images and photogrammetric tools is around three months before the harvest. No relationship was found between photogrammetric estimations and other sugar content variables of sugarcane.

Las aplicaciones de herramientas geoespaciales en cultivos son variadas, aunque coinciden en la necesidad de comprender la variabilidad espacial y temporal implícita en las áreas de producción agrícola, a partir de la construcción de modelos que tomen en cuenta esta variabilidad (Molin y Veiga 2016). Basadas en los Sistemas de Información Geográfica (SIG) y Teledetección, estas metodologías proveen una mayor versatilidad para el manejo de información de fenómenos espacialmente distribuidos (Chuvieco 2008). Por ejemplo, la fotogrametría con el uso de VANT complementadas 
con cámaras RGB y/o multiespectrales, han facilitado el acceso a información cada vez más precisa y rápida de las plantaciones agrícolas (Hoyos y Fritchi 2013). Algunos ejemplos han estado relacionados a información del estado fitosanitario de las plantas, estimaciones del crecimiento de los cultivos, biomasa, estimaciones de cosechas, determinación de condiciones de estrés, entre otras aplicaciones (García y Montero 2016, Chong et.al. 2017).

En Costa Rica, el cultivo de caña de azúcar es una actividad agrícola tradicional y de gran relevancia, no solo por aspectos económicos y sociales, sino también por su extensión en el país, así como los recursos naturales que demanda su producción, como el agua y el suelo (MAG 2007). Por tanto, toda investigación que permita mejorar las estrategias, enfocadas al aumento en la productividad, tiene gran relevancia para el sector azucarero, debido a la presión en el uso de los recursos y la demanda de insumos. Existe la necesidad de optimizar los mecanismos que permitan evaluar y monitorear el comportamiento del cultivo durante los ciclos de crecimiento, lo cual se puede hacer a través de diversas metodologías para el monitoreo de sistemas agrícolas. Lo anterior permitiría dar una respuesta pronta y oportuna a las personas productoras ante los fenómenos que suceden dentro de los campos agrícolas (Raj et al. 2020).

García y Montero (2016) evaluaron el uso de imágenes satelitales y fotografías obtenidas con VANT para estimar la productividad en caña de azúcar a partir de índices de vegetación; además, utilizaron los datos del Modelo de Digital de Superficie (DSM por sus siglas en inglés) y el Modelo de Elevación Digital (DEM por sus siglas en inglés) para calcular la altura promedio y relacionarla con la productividad obtenida, logrando obtener un $\mathrm{R}^{2}$ de 0,43 cuando el cultivo tenía 6 meses de plantado.

En otros estudios, se utilizó la información de reflectancia de imágenes satelitales, para el desarrollo de otras aplicaciones, como fue la estimación de sacarosa en caña de azúcar. También, correlacionar los datos de biomasa y sacarosa en una serie de tiempo, permitió monitorear los requerimientos del cultivo en cuanto al estado nutricional, requerimientos hídricos y otros (Zhao et al. 2016, Rahman 2008).

Estas herramientas además permiten dar seguimiento y monitoreo a los cultivos en los diferentes estados fenológicos mediante estimados de las características biométricas de las plantaciones (García y Herrera 2015). Por ejemplo, con la utilización de los DSM y los DEM, generados con diferentes cámaras instaladas en un VANT, es posible calcular la altura de los cultivos, lo que genera un insumo de mucha utilidad para el mejoramiento y la gestión de los factores bióticos y abióticos que influyen desarrollo de los cultivos (Lillesand y Kiefer 1987, García y Herrera 2015). Luego, a partir de estas estimaciones biométricas, es posible realizar estimaciones de productividad, que permitan definir estrategias de manejo de acuerdo con el comportamiento espacial y temporal del cultivo (Furukawa et al. 2020).

El concepto se basa en utilizar técnicas fotogramétricas que contribuyan a cuantificar dimensiones y formas del terreno a partir de fotografías aéreas (Redweik 2013, Bending et al. 2014). Esta técnica comprende un conjunto de métodos, procedimientos y tecnologías, como VANT y software especializados, que ayudan a obtener medidas precisas de distancias y elevaciones de la superficie terrestre a partir de fotografías, por medio de parámetros de corrección los ángulos y la medición de las coordenadas XY en las fotografías, asociadas a las coordenadas del terreno (X, Y, y Z) además, de la incorporación de puntos de control identificados en el terreno y en las fotografías, lo cual permite corregir la geometría de los objetos mapeados con bastante detalle (Lillesand y Kiefer 1987,Campbell 2006, Redweik 2013).

El objetivo de esta investigación fue estimar las variables de rendimiento TMH, TKA, RKA, TKM y RKM en una plantación de caña de azúcar, mediante el cálculo de la altura obtenida a partir de DSM y DEM, calculados con fotografías aéreas obtenidas con VANT. 


\section{MATERIALES Y MÉTODOS}

La investigación se llevó a cabo en un lote de la cooperativa CoopeCañita R.L, ubicado en el cantón de Turrialba, Costa Rica entre las coordenadas $9,847107^{\circ}-9,847802^{\circ}$ latitud Norte y $83,672923^{\circ}-83,671532^{\circ}$ longitud Oeste. El lote tenía una extensión aproximada de una hectárea y fue subdividido en 10 sublotes, en cada uno de los cuales se hizo la evaluación de las variables de rendimiento de campo a nivel comercial de caña de azúcar. Esta subdivisión se realizó para facilitar el tratamiento de la información y para obtener más fuentes de variación de crecimiento del cultivo y sus correspondientes variables de rendimiento, así como, el disminuir la variabilidad espacial que pudiese afectar el análisis final de los datos (Figura 1).

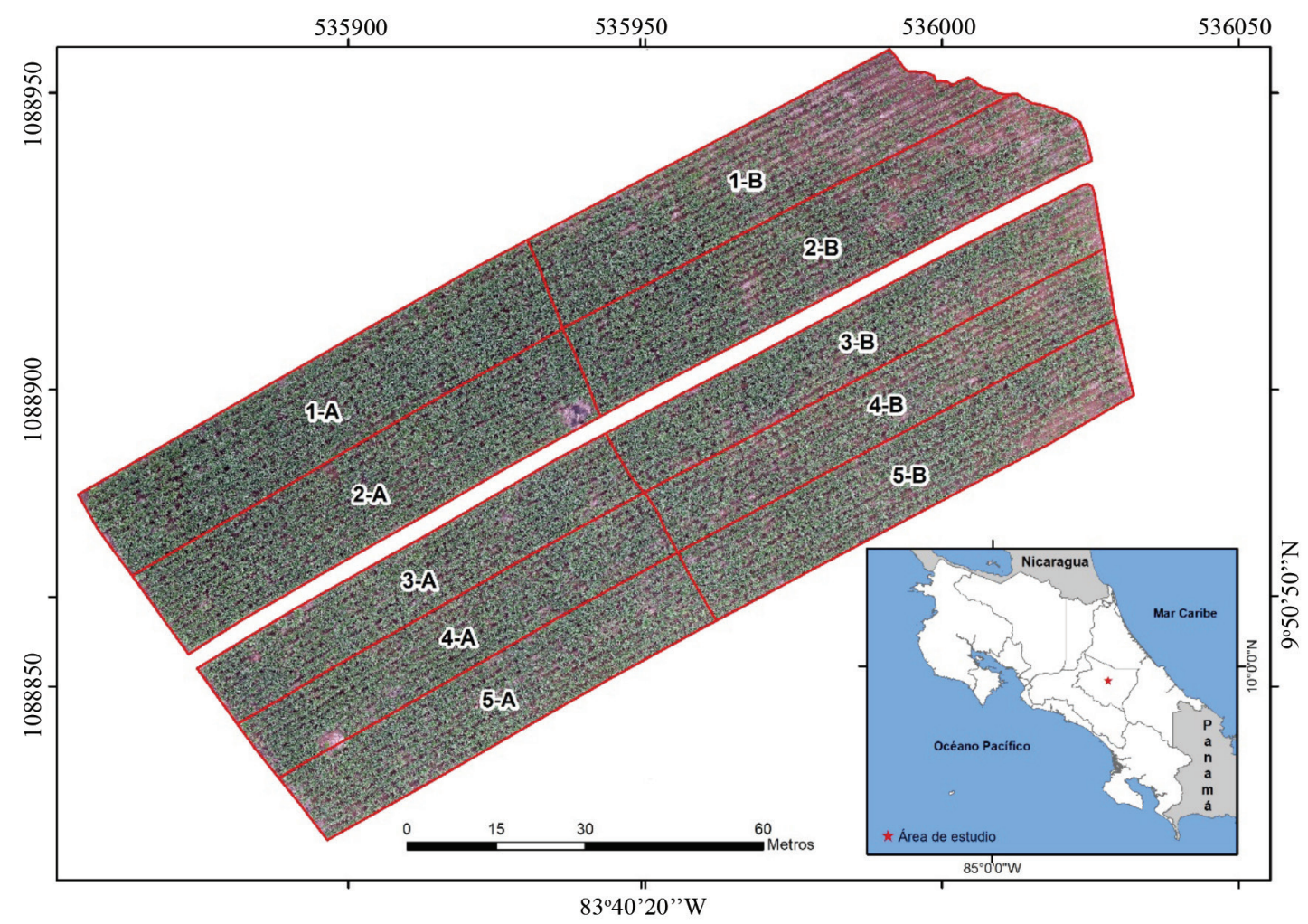

Figura 1. División de los sublotes en el lote "La Palma", Turrialba. Costa Rica. Los sublotes A representan áreas de mejor crecimiento y los sublotes B identifican áreas de crecimiento deficiente.

Durante los vuelos, se identificó visualmente que los sublotes A presentaron un mejor crecimiento que los sublotes B, por tal razón, se decidió separar los datos de producción durante la cosecha, para evitar la generalización de la información; la separación de los sublotes de 1 a 5 se realizó para aumentar el número de registros (repeticiones).
El lote estuvo sembrado con la variedad de ciclo anual B76-259 en su quinto año de producción. Esta variedad es ampliamente sembrada y distribuida en toda la región (Chávez et al. 2001) y tiene como principales características biotípicas el crecimiento erecto en los primeros meses de desarrollo, tallos de grosor medio, excelente despaje natural, 
precoz (ciclo de 12 meses) y con una alta capacidad potencial de concentrar azúcar, que supera usualmente los 100 ton.ha $^{-1}$ de azúcar (Calderón y Chávez 2003).

La cosecha fue realizada en febrero de 2018 por los colaboradores de la Cooperativa, mediante la separaración de producto en cada sublote. Las variables de producción obtenidas durante la cosecha fueron:

- $\quad$ Toneladas métricas por hectárea $(\mathrm{TMH})$ : este valor corresponde al total de toneladas cosechadas, en cada sublote, dividido por el área del sublote.

- Rendimiento de kilogramos de azúcar (RKA): es el valor promedio de los kilogramos de azúcar que se obtuvieron en cada tonelada cosechada por sublote.

- $\quad$ Total de kilogramos de azúcar (TKA): es la suma del total kilogramos de azúcar obtenidos en los distintos sublotes.

- $\quad$ Total de kilogramos de miel (TKM): es la suma del total kilogramos de miel obtenidos en cada sublote.

Rendimiento de kilogramos de miel (RKM): es el valor promedio de los kilogramos de miel obtenidos en cada tonelada cosechada por sublote.
De mayo a noviembre del 2017, se realizaron 6 vuelos con un VANT modelo Aibot X6V2 que portaba una cámara Nikon CoolPix A de 16,2 megapíxeles de resolución. En cada vuelo se tomaron fotografías, que cubrían la totalidad del área de interés, todos los sublotes quedaban cubiertos en un único vuelo. El proceso de alineamiento y ortorectificación de las fotografías obtenidas, en cada vuelo, se realizaron con el software Agisoft Photoscan, el cual permitió la elaboración de un único mosaico que cubría la totalidad de los sublotes del área de estudio. Las fotografías tuvieron un traslape frontal de $80 \%$ y un traslape lateral de $70 \%$. Estos traslapes se obtuvieron a partir de la programación del vuelo en el software Aibotix AiProFlight 2.9, en el cual se definió la altura de vuelo de 70 metros y los traslapes mencionados, es importante resaltar que durante todos los vuelos se utilizó el mismo plan de vuelo. Además, se realizó una corrección geométrica del ortomosaico con puntos de control de campo que fueron obtenidos con una estación total marca GeoMax.

En total se colocaron 13 puntos de control que fueron ubicados perimetralmente y en la calle central que atravesaba el área de estudio (Figura 2). El uso de puntos de control permitió obtener DSM con errores promedio de posicionamiento inferiores a los $5 \mathrm{~cm}$ en los ejes X,Y,Z.

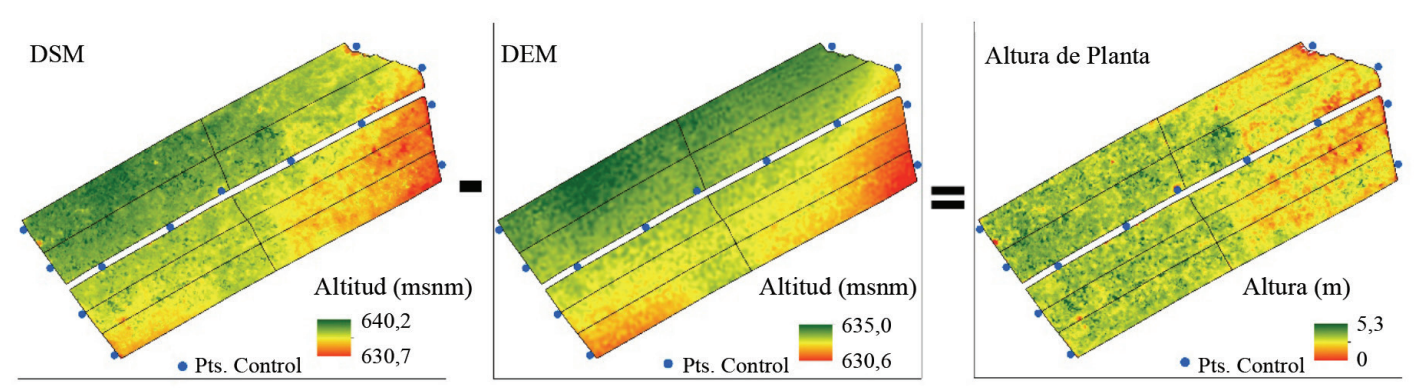

Figura 2. Procedimiento para calcular la altura de planta (por pixel) a partir del DSM y el DEM con la calculadora ráster. Además, se presenta la ubicación de los puntos de control en la corrección geométrica de los modelos.

Se decidió hacer los vuelos hasta noviembre, aproximadamente 3 meses antes de la cosecha, en el entendido de que esta variedad de caña inicia el despaje natural al tiempo que aumenta el número de plantas volcadas, comportamiento propio de la variedad, alrededor de 90 días antes 
de cosecha. Este hecho, además, fue corroborado a nivel de campo. El haber incluido DSM en esa etapa final de la plantación, hubiese ocasionado un retroceso en la altura calculada.

Se utilizó el DEM generado como base para comparar el crecimiento del cultivo en cada una de las evaluaciones. Dicho modelo se obtuvo cuando aún los rebrotes de caña eran mínimos y podían ser desestimados en el DEM. De esta manera, el DEM generado sirvió como referencia y comparación contra el DSM obtenido en cada evaluación. Al realizar la resta de los ráster DSM menos DEM, se obtiene la altura en cada uno de los pixeles (con resolución espacial de $0,005 \mathrm{~m}$ ) de área de estudio de la siguiente manera (Figura 2).

$$
A P=D S M-D E M
$$

Donde:

AP = Altura de la planta en cada uno de los pixeles

DSM $=$ Modelo digital de superficie para un mes determinado

$\mathrm{DEM}=$ Modelo de elevación digital

Una vez obtenido el ráster de altura del área de estudio, se procedió a calcular el volumen (este valor puede ser convertido a biomasa, sin embargo, en este trabajo no se contaba con el valor de la masa que permitía realizar la conversión) en cada uno de los pixeles, mediante siguiente fórmula:

$$
V=A P X 0,0025 \mathrm{~m}^{2}
$$

Donde:

$$
\begin{aligned}
& \mathrm{AP}=\begin{array}{l}
\text { Altura de la planta en cada uno de los } \\
\text { pixeles }
\end{array} \\
& 0,0025 \mathrm{~m}^{2}=\text { Tamaño del píxel del ráster }
\end{aligned}
$$

Posteriormente, se extrajo el promedio de altura y el volumen obtenido de cada sublote en las 6 evaluaciones mediante la herramienta Zonal Statistics de ArcGIS 10.5. Las variables de altura y volumen estimadas que fueron obtenidos a partir del proceso anterior, se correlacionaron con la información de producción de la zafra de ese año (2017-2018), que se obtuvo en cada uno de los sublotes.

A partir de la altura y el volumen estimados fotogramétricamente y los datos de cosecha de campo, se construyeron modelos lineales para validar el poder predictivo de la variable altura del cultivo sobre el dato real de cosecha.

\section{RESULTADOS Y DISCUSIÓN}

Análisis espacio-temporal de crecimiento del cultivo. Al evaluar el comportamiento del crecimiento en altura del cultivo de la caña de azúcar por métodos fotogramétricos con el uso del VANT, se identificaron diferencias entre los sublotes desde el inicio de las evaluaciones. Estas diferencias fueron más evidentes conforme aumentaba la edad de la plantación, lo cual coincide con lo planteado por Sofonia et al. (2019), porque presentó un crecimiento bastante limitado, comparado a los otros lotes que presentaron siempre un mayor crecimiento, el cual se mantuvo a través de los meses y durante las 6 evaluaciones (Figura 3). El bajo crecimiento se debió a las condiciones particulares del suelo y no afectaron los resultados, más bien aportaron datos variables que enriquecieron el estudio.

Estas diferencias se hicieron más notorias en la última evaluación; los sublotes de la zona A presentaron un promedio de altura de $3,2 \mathrm{~m}$, en tanto los sublotes de la zona B con un menor crecimiento presentaron una altura promedio de 2,5 m (Figura 3). 


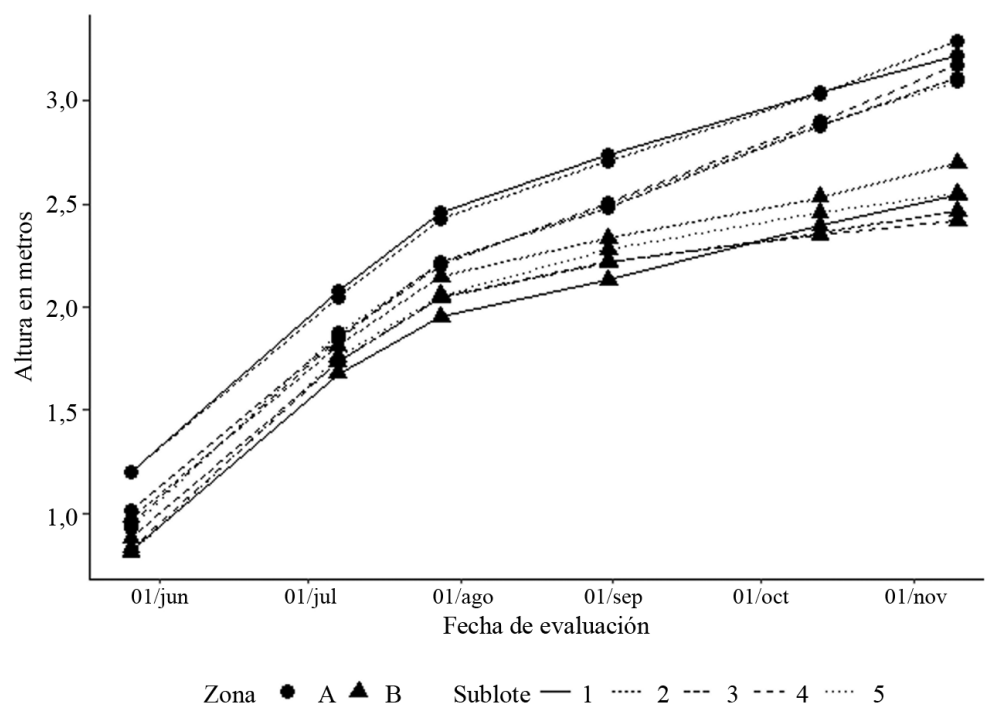

Figura 3. Promedio del crecimiento de caña de azúcar por sublote, en los 10 sublotes durante las 6 evaluaciones (26/06/2017, 07/07/2017, 28/07/2017, 31/08/2017, 13/10/2017 y 10/11/2017). Existe una evidente separación entre los sublotes de la zona A con respecto a la zona B, principalmente a partir de 28/07/2017.

De manera general, el comportamiento espacio-temporal, del crecimiento del cultivo, presentó 2 tendencias: Los sublotes en la zona $B$ presentaron un menor promedio de altura de planta durante todas las evaluaciones, caso contrario sucedió con los sublotes de la zona A, que presentaron los mayores promedios de altura, esa tendencia se reflejó en la variabilidad espacial y temporal de la altura de las plantas (Figura 4). 

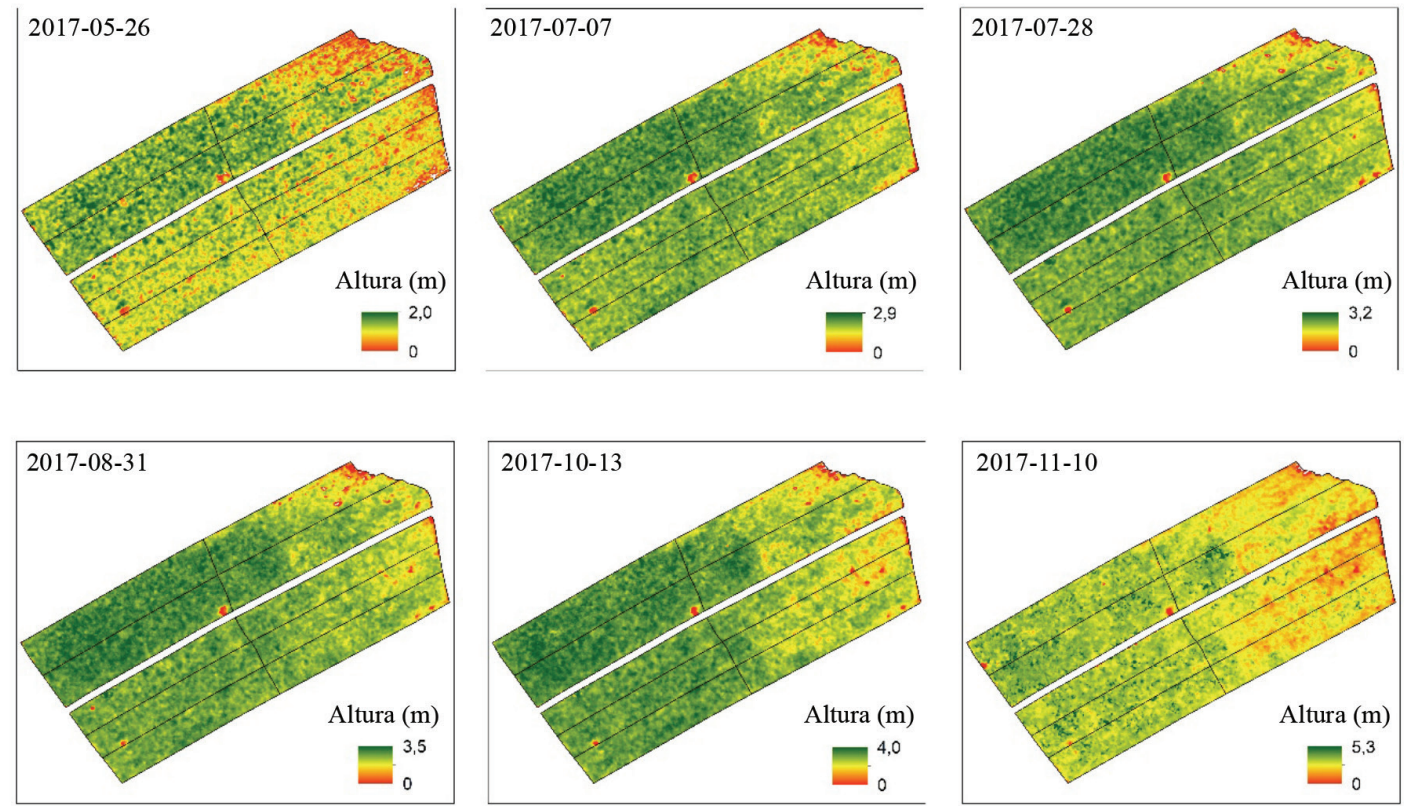

Figura 4. Variación espacio-temporal de la altura de las plantas de caña de azúcar en los sublotes del área de estudio. Estimación de altura por fotogrametría los días 26/06/2017, 07/07/2017, 28/07/2017, 31/08/2017, 13/10/2017 y 10/11/2017.

En relación con el cálculo del volumen, se encontró la misma tendencia que la altura, al compararlo espacial y temporalmente con las variables de rendimiento. Esto porque el volumen proviene de multiplicar la altura de la planta por un mismo factor (Írvem 2011), el cual corresponde al área de cada pixel $(0,0025$ $\mathrm{m}^{2}$ ), por lo que era esperable que existiera una colinealidad entre la altura y el volumen de la biomasa de las plantas, de donde se presentó una completa similitud en el comportamiento espacio temporal de ambas variables con las variables de rendimiento. Debido a lo anterior, se utilizó únicamente la altura de planta para realizar las correlaciones y las estimaciones con las variables de rendimiento.

Relación entre el promedio de altura de plantas y las variables de rendimiento. $\mathrm{Al}$ establecer el análisis de correlación entre el promedio de altura obtenido, en cada una de las
6 determinaciones y las variables de rendimiento de campo obtenidas en la zafra, se lograron obtener relaciones positivas y congruentes con lo observado a nivel de campo, lo cual confirma que la altura de planta podría ser un buen indicador del cultivo, porque está relacionado variables contenido de nitrógeno en hojas y biomasa (Portz et al. 2012).

En primer lugar, se encontró que el valor de correlación entre las variables de rendimiento y la altura fue en aumento, conforme se incrementó la edad del cultivo (Sofonia et al. 2019), en otras palabras, la altura y el volumen de plantas al inicio del ciclo fenológico expuso muy baja correlación con las variables de rendimiento, debido a la ausencia de área foliar (Figura 5), lo que validó el hecho de que las mejores determinaciones serían cuando el dosel estuviera cerrado (Sanches et al. 2018); además, las mejores correlaciones se alcanzaron al final de las evaluaciones. Debido a lo anterior, el uso de esta metodología de 
sensoramiento remoto para estimar variables de rendimiento, tendrá sentido si se utiliza en la etapa de crecimiento máximo (en el mes de noviembre para el caso de este estudio). Rahman y Robson (2020) definieron la etapa de máximo crecimiento como la mejor fecha para la estimación de toneladas métricas de caña de azúcar por hectárea, Mutanga et al. (2013) determinaron 2 meses antes de cosecha como el mejor momento para realizar estimaciones con imágenes satelital. Si no es este el objetivo, una alternativa sería hacer monitoreos estacionales o bien monitoreos de seguimiento a tratamientos aplicados en etapas iniciales de crecimiento (Sofonia et al. 2019).

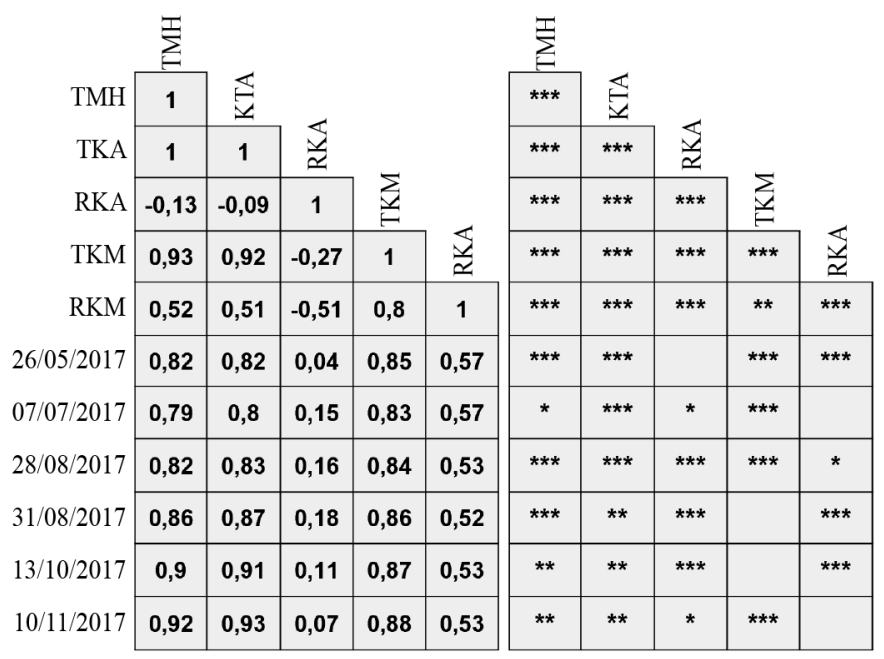

Figura 5. Correlación de Pearson y niveles de significancia $(* * *<0,001, * *<0,01 \mathrm{y} *<0,05)$ entre el promedio de altura en cada una de las evaluaciones (estimado mediante DSM y DEM) y las variables de rendimiento (TMH, TKA, RKA, TKM y RKM).

Se encontró que la mayor correlación fue realizada en la determinación del 10/11/2017, en la cual las variables de rendimiento TMH, TKA y TKM, presentaron las mejores correlaciones (valor de "R") con respecto al promedio de altura (0,91, 0,92 y 0,88, respectivamente). La correlación de 0,91 , entre la altura de plantas y el rendimiento en $\mathrm{TMH}$, fue superior a la encontrada por Mutanga et al. (2013) al correlacionar las mismas variables ("R" de 0,58).

A diferencia de lo encontrado por Rahman (2008), otras variables de rendimiento RKA y RKM presentaron muy bajas correlaciones (valor de "R") con el promedio de altura $(0,33$ y 0,44 , respectivamente), lo cual confirmó que la altura de las plantas es una variable que tiene baja relación con la eficiencia o el rendimiento de kilogramos de azúcar por tonelada (Shukla et al. 2017) (Figura 5).

Con el fin de modelar la alta correlación obtenida entre las variables TMH, TKA y TKM con la altura de plantas en la última evaluación, se crearon un conjunto de modelos lineales que pretendían relacionar los rendimientos del cultivo de la caña de azúcar para este caso en particular, en función del promedio de altura. Los resultados se presentan en la Tabla 1. 
Tabla 1. Modelos lineales obtenidos al analizar las variables de rendimiento TMH, TKA, RKA, TKM y RKM en función del promedio de altura estimado para la evaluación de noviembre.

\begin{tabular}{lccc}
\hline Modelo & Error residual estándar & $\mathrm{R}^{2}$ ajustado & $\mathrm{p}$-value \\
\hline $\mathrm{TMH}=40,993 *$ Altura $-48,829$ & 6,296 & 0,836 & 0,0001314 \\
$\mathrm{TKA}=4481 *$ Altura $-5362,4$ & 648,2 & 0,8521 & $8,63 * 10-05$ \\
$\mathrm{RKA}=0,2258 *$ Altura $-108,33$ & 1,149 & 0,119 & 0,8414 \\
$\mathrm{TKM}=1574,4 *$ Altura -2573 & 319,5 & 0,7705 & 0,000840 \\
$\mathrm{RKM}=6,093 *$ Altura $-10,352$ & 3,633 & 0,19 & 0,1158 \\
\hline
\end{tabular}

Como se puede observar, los modelos lineales confirmaron que las variables RKA y RKM presentaron bajas correlaciones con el promedio de la altura (y por ende, por la colinealidad mencionada anteriormente, con el volumen estimado). Por el contrario, las variables con mejores coeficientes de determinación ajustados fueron TMH y TKA con un $84 \%$ y un $85 \%$, respectivamente, y en menor grado TKM con un $77 \%$.

Lo anterior, sugiere que no necesariamente mayores alturas de plantas están relacionadas a mayores concentraciones de azúcar por área (Shukla et al. 2017) por lo que sería bueno estudiar la integración de otros medios de determinación remota que sean más sensibles a esta variable.

La ejecución del modelo de estimación TMH permitió determinar que los sublotes 1-A, 1-B, 2-A, 3-A y 3-B presentaron los menores errores absolutos de estimación, con errores de

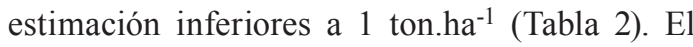
sublote con mayor error absoluto de estimación fue 5-A con un error de 12,6 ton.ha-1 (Tabla 2). El promedio general de error absoluto fue 3,7 ton.ha ${ }^{-1}$. La información de la Tabla 2 permitió validar la correcta ejecución del modelo TMH.

Tabla 2. Evaluación de la estimación de TMH utilizando la función TMH $=40,993 * A 1 t u r a-48,829$.

\begin{tabular}{ccccc}
\hline Sublote & TMH real & TMH estimado & Error de estimación & $\begin{array}{c}\text { Error absoluto de } \\
\text { estimación }\end{array}$ \\
\hline 1-A & 84,0 & 83,1 & $-0,9$ & 0,9 \\
1-B & 55,0 & 55,3 & 0,3 & 0,3 \\
2-A & 85,9 & 86,0 & 0,1 & 0,1 \\
2-B & 67,4 & 61,6 & $-5,7$ & 5,7 \\
3-A & 77,9 & 78,7 & 0,8 & 0,8 \\
3-B & 52,8 & 52,2 & $-0,6$ & 0,6 \\
4-A & 91,0 & 81,1 & $-10,0$ & 10,0 \\
4-B & 51,7 & 50,2 & $-1,4$ & 1,4 \\
5-A & 65,4 & 78,0 & 12,6 & 12,6 \\
5-B & 50,8 & 55,6 & 4,8 & 4,8 \\
\hline
\end{tabular}


El cálculo de la raíz cuadrada del error cuadrático medio (RMSE, por sus siglas en inglés), para el modelo TMH, fue de 5,63 ton. ha $^{-1}$, este resultado también fue similar al obtenido por Luna y Lobo (2016) que al utilizar VANT para estimar TMH obtuvieron un RMSE de 5,04 ton.ha-1. Rahman y Robson (2020) obtuvieron un RMSE 11,33 ton.ha-1 al estimar la cantidad de toneladas métricas por hectárea por medio de índices de vegetación calculados a partir de imágenes satelitales.
Por medio de los modelos de estimación que se presentan en el Tabla 1, se estimó la variabilidad espacial únicamente de las variables de rendimiento TMH, TKA y TKM, porque presentaron resultados más robustos. En la Figura 6 , es posible observar la variación espacial de cada variable de rendimiento, este resultado es muy congruente con la variación espacial de la altura de las plantas, donde las variables de rendimiento presentaron determinaciones inferiores en la zona B. 

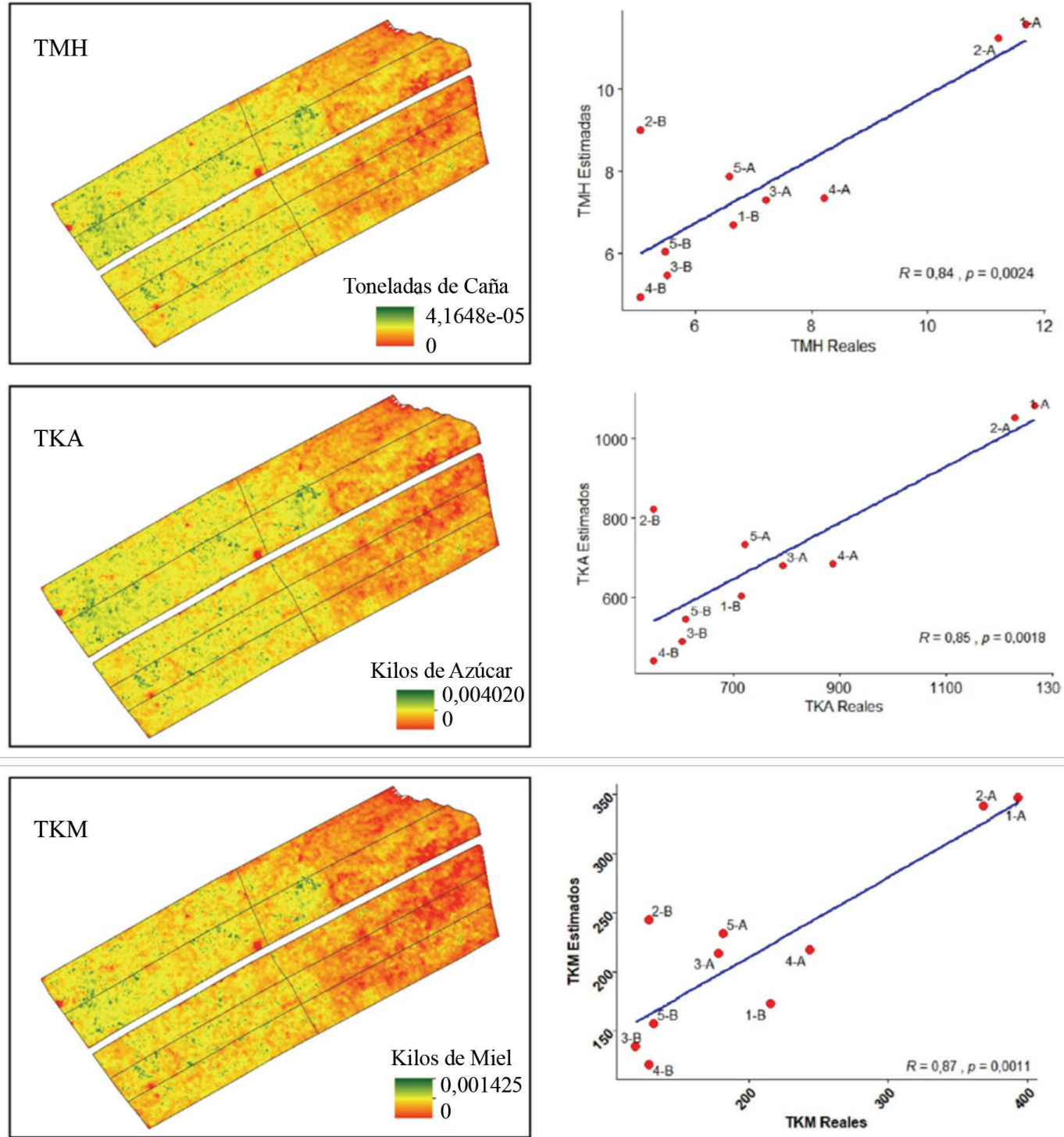

Figura 6. Estimación de las variables TMH, TKA y TKM a partir de los modelos lineales de la Tabla 1. Los mapas de la izquierda muestran la estimación de las variables para el área del pixel.

Los gráficos de la derecha presentan la correlación de Pearson entre la variable evaluada en campo y la variable estimada a partir de la sumatoria de todos los pixeles que conforma cada sublote.

Con base en los datos obtenidos, se pudo determinar que las correlaciones entre las variables estimadas por el método fotogramétrico y los reales fueron altas, aspecto que sugiere que la aplicación de esta metodología tiene buenos niveles de acierto, esto lo confirma el bajo RMSE, con potencial para convertirse en una herramienta para el cultivo de la caña de azúcar. 


\section{CONCLUSIONES}

Con la utilización de sensores remotos transportados en un VANT, fue posible obtener los DSM y DEM con los cuales fue posible hacer estimaciones de altura de cultivo y volumen de una plantación de caña de azúcar; estos a su vez, correlacionaron positivamente con las variables de rendimiento de campo, lo que sugiere que la herramienta metodológica planteada puede constituirse en una vía importante para hacer estimaciones de rendimiento en el cultivo de la caña de azúcar de forma remota y previo a la cosecha (el $\mathrm{R}^{2}$ para los últimos 2 meses fue superior a 0,8$)$. A pesar de las buenas expectativas con esta metodología, el rendimiento de azúcar, por tonelada, deberá ser evaluado con el uso de otros procedimientos debido a la baja correlación encontrada. Estos otros métodos por valorar con más detenimiento, incluirían por ejemplo, el uso de cámaras multiespectrales, información que puede ser complementada con las herramientas utilizadas en este estudio. No hay duda de que estos modelos de estimación se pueden convertir en insumos de gran potencial para optimizar procesos productivos y favorecer la toma de decisiones en el sector azucarero, que en la actualidad requiere mejorar la competitividad.

\section{AGRADECIMIENTOS}

Las personas autoras externan un profundo agradecimiento a las personas colaboradoras de INFOCOOP y CoopeCañita R.L. que apoyaron el desarrollo de esta investigación.

\section{LITERATURA CITADA}

Bending, J; Bolten, A; Bennertz, S; Broscheit, J; Eichfuss, S; Bareth, G. 2014. Estimating Biomass of Barley Using Crop Surface Models (CSMs) Derived from UAV-Based RGB Imaging. Remote Sensing 6(11):10395-10412.

Calderón, G; Chávez, M. 2003. Estudio agroindustrial de cuatro densidades de siembra con la variedad de caña de azúcar b76-259 cultivada en Atirro, Turrialba. Promedio de tres cosechas. In Congreso
ATACORI (1, 2003, Guanacaste, Costa Rica). Memoria. Costa Rica. p. 251-255.

Campbell, J. 2006. Plant Sciences. Introduction to remote sensing. New York, Estados Unidos. 447 p.

Chávez, M; Rodríguez, M; Villalobos, C; Angulo, A; Calderón G; Alfaro, R; Rodríguez, J. 2001. Censo de variedades de caña de azúcar de Costa Rica año 2000. San José, Costa Rica. 45 p.

Chong, KL; Kanniah, KD; Pohl, C; Tan, KP. 2017. A review of remote sensing applications for oil palm studies. Geo-spatial Information Science 20(2):184-200.

Chuvieco, E. 2008. Teledetección ambiental. 2 ed. Barcelona, España. Ariel. 594 p.

Furukawa, F; Maruyama, K; Saito, Y; Kaneko, M. 2020. Corn Height Estimation Using UAV for Yield Prediction and Crop Monitoring. In Avtar, R; Watanabe, T. (eds.). Unmanned Aerial Vehicle: Applications in Agriculture and Environment. Suiza, Springer. p. 51-69.

García, C; Herrera, F. 2015. Percepción remota en cultivos de caña de azúcar usando una cámara multiespectral en un vehículo aéreo no tripulado. In Anais Simposio Brasileiro de sensoramiento remoto-SBSR (17, 2015, João Pessoa-PB, Brasil). Memoria. Brasil. p. 4450-4457.

García, C; Montero, D. 2016. Modelo de Aforo de caña de azúcar a partir de modelos digitales de altura e índices de vegetación. In Simposio Internacional en Percepción Remota y Sistemas de Información Geográfica (17, 2016, Puerto Iguazú Misiones, Argentina). Memoria. Argentina. p. 1199-1208.

Hoyos, V; Fritchi, F. 2013. Relationships Among Vegetation Indices Derived from Aerial Photographs and Soybean Growth and Yield. Crop Science 53:2631-2642.

Írvem, A. 2011. Application of GIS to Determine Storage Volume and Surface Area of Reservoirs: The Case Study of Buyuk Karacay Dam. International Journal of Natural and Engineering Sciences 5(1):39-43.

Lillesand, TM; Kiefer, RW. 1987. Remote sensing and image interpretation. 2 ed. New York, Estados Unidos. John Wiley \& Sons. 721 p.

Luna, I; Lobo, A. 2016. Mapping Crop Planting Quality in Sugarcane from UAV Imagery: A Pilot Study in Nicaragua. Remote Sensing 8(6):2-18.

MAG (Ministerio de Agricultura y Ganadería). 2007. Agrocadena de la caña de azúcar para la producción de dulce. Puriscal, Costa Rica. 80 p.

Marcos. JL; Gil, M; Ortiz, J; Martínez, S; Garrido, F; Sánchez, L; Hernández, S; Clérigo, Z; Ortiz, L. 2016. Determinación de biomasa en parcelas de cultivos herbáceos mediante cámaras ópticas elevadas por medio de vehículos aéreos no tripulados (UAV). In Congreso Iberoamericano de Investigación Cualitativa en Ingeniería y Tecnología $(5,2016$, Oporto, Portugal). Memoria. Portugal 4:95-103. 
Mutanga, S; Schoor, C; Olorunju, P.L; Gonah, T; Ramoelo, A. 2013. Determining the Best Optimum Time for Predicting Sugarcane Yield Using Hyper-Temporal Satellite Imagery. Advances in Remote Sensing 2(03):269-275.

Molin, J; Veiga, J. 2016. Spatial variability of sugarcane row gaps: measurement and mapping. Ciência e Agrotecnología 40 (3):347-355.

Oshunsanya, S; Aliku, O. 2016. GIS Applications in Agronomy. In Imperatone, P; Pepe, A (eds.). Geospatial Technology - Environmental and Social Applications. Roma, Italia. IntechOpen. p. 217-234.

Portz, G; Amaral, LR; Molin, JP. 2012. Measuring Sugarcane Height in Complement to Biomass Sensor for Nitrogen Management. In International Conference on Precision Agriculture (10, 2012, Indianapolis, Estados Unidos). Memoria. Estados Unidos. 10 p.

Raj, R; Kar, S; Nandan, R; Jagarlapudi, A. 2020. Precision agriculture and unmanned aerial Vehicles (UAVs). In Avtar, R; Watanabe, T. (eds.). Unmanned Aerial Vehicle: Applications in Agriculture and Enviroment. Suiza. Springer. p. 7-23.

Rahman, E. 2008. The application of remote sensing techniques to sugarcane (Saccharum spp. hybrid) production: A review of literature. International Journal of Remote Sensing 29(13):3753-3767.

Rahman, M; Robson, A. 2020. Integrating Landsat-8 and Sentinel-2 Time Series Data for Yield Prediction of Sugarcane Crops at the Block Level. Remote Sensing 12(8):1-15.
Redweik, P. 2013. Photogrammetry. In Gouchang, X. (eds.) Science of Geodesy-II Innovation and Future Developments. Alemania. Springer. p. 133-183.

Sanches, GM; Duft, DG; Kölln, OT; Luciano, AC; De Castro, SG; Okuno, FM; Franco, HC. 2018. The potential for RGB images obtained using unmanned aerial vehicle to assess and predict yield in sugarcane fields. International Journal of Remote Sensing 39(15-16):5402-5414.

Shunling, L. 2004. Quantitative Remote Sensing of land surfaces. New Jersey, Estados Unidos, John Wiley \&Sons. 562 p.

Sofonia, J; Sheldryk, Y; Phinn, S; Roelfsema, C; Kendoul, F; Skocaj, D. 2019. Monitoring sugarcane growth response to varying nitrogen application rates: A comparison of UAV SLAM LiDAR and photogrammetry. Int $\mathrm{J}$ Appl Earth Obs Geoinformation 82:1-15.

Som-ard, J; Dalower, M; Ninsawat, S; Veerachitt, V. 2018. Pre-harvest Yield Estimation Using UAV-Based RGB Imeges and Ground Observartion. Sugar Tech. 20(3):645-657.

Shukla, S; Felderhoff, T.J; Saballos, A; Vermerris, W. 2017. The relationship between plant height and sugar accumulation in the stems of sweet sorghum (Sorghum bicolor (L.) Moench). Field Crops Research 203:181-191.

Zhao, Y; Della, D; Kazama, Y; Vieira, J; Graziano, P; Camargo, R. 2016. Dynamics modeling for sugarcane sucrose estimation using time series satellite imagery. Remote Sensing for Agriculture, Ecosystems, and Hydrology 18:1-11. 